\title{
Super LeArner Prediction of NAb Panels (SLAPNAP): A Containerized Tool for Predicting Combination Monoclonal Broadly Neutralizing Antibody Sensitivity
}

Benkeser D*, Department of Biostatistics and Bioinformatics, Emory University, Atlanta, GA 30322, USA

Williamson BD*, Vaccine and Infectious Disease Division, Fred Hutchinson Cancer Research Center, Seattle, WA 98109, USA

Magaret CA, Vaccine and Infectious Disease Division, Fred Hutchinson Cancer Research Center, Seattle, WA 98109, USA

Nizam S, Department of Biostatistics and Bioinformatics, Emory University, Atlanta, GA 30322, USA

Gilbert PB, Vaccine and Infectious Disease and Public Health Sciences Divisions, Fred Hutchinson Cancer Research Center, Seattle, WA 98109, USA; Department of Biostatistics, University of Washington, Seattle, WA 98195, USA

*authors contributed equally to this manuscript

\section{Abstract}

Summary: Single broadly neutralizing antibody (bnAb) regimens are currently being evaluated in randomized trials for prevention efficacy against HIV-1 infection. Subsequent trials will evaluate combination bnAb regimens (e.g., cocktails, multi-specific antibodies), which demonstrate higher potency and breadth in vitro compared to single bnAbs. Given the large number of potential regimens in the research pipeline, methods for down-selecting these regimens into efficacy trials are of great interest. To aid the down-selection process, we developed Super LeArner Prediction of NAb Panels (SLAPNAP), a software tool for training and evaluating machine learning models that predict in vitro neutralization resistance of HIV Envelope pseudoviruses to a given single or combination bnAb regimen, based on Envelope amino acid sequence features. SLAPNAP also provides measures of variable importance of sequence features. These results can rank bnAb regimens by their potential prevention efficacy and aid assessments of how prevention efficacy depends on sequence features.

Availability and Implementation: SLAPNAP is a freely available docker image that can be downloaded from DockerHub (https://hub.docker.com/r/slapnap/slapnap). Source code and documentation are available at GitHub (respectively, https://github.com/benkeser/slapnap and https://benkeser.github.io/slapnap/).

Contact: David Benkeser, benkeser@emory.edu

\section{Key Words}

Broadly neutralizing antibodies, HIV-1, sieve analysis, efficacy trials, superlearning, variable importance estimation, proteomic antibody resistance score, ranking and down-selection, combination regimen, multi-specific regimen 
Extensive research has been conducted on passive administration of monoclonal broadly neutralizing antibodies (bnAb) with the objective of preventing HIV-1 infection (Morris and Mkhize, 2017). BnAbs target conserved epitopes on the HIV-1 envelope (Env) glycoprotein, and recent work has shown that combination bnAb regimens can neutralize most clinical HIV-1 isolates in genetically diverse Env panels [e.g., (McCoy and Burton, 2017; Sok and Burton, 2018)]. BnAb regimens have also been shown to prevent SHIV infection in nonhuman primate models (Gautam et al., 2016; Hessell et al., 2018; Pegu et al., 2019). These developments position bnAbs as promising tools for the prevention of HIV-1, as well as other infectious diseases, in the near future (Karuna and Corey, 2020).

A key issue in developing efficacious bnAb regimens is understanding the neutralization breadth and potency of a given bnAb regimen against HIV-1 Env panels that are representative of circulating virus populations (Wagh et al., 2016; Wagh et al., 2018). Several bnAb combinations targeting distinct Env epitopes have been identified that exhibit greater neutralization breadth and potency than their constituent single bnAbs, with in vitro neutralization coverage rates approaching 100\% (Doria-Rose et al., 2012; Kong et al., 2015; Wagh et al., 2016). Thus, while the trials that are furthest advanced in the clinical pipeline [the Antibody Mediated Prevention trials (Gilbert et al., 2017)] are evaluating the prevention efficacy of a single passively administered bnAb, VRC01, future trials will likely focus on bnAb combination regimens or multi-specific bnAb regimens. Several such early-phase clinical trials are being planned or are underway (e.g., NCT04212091, NCT03928821). 
Analysis of efficacy trials can elucidate in vivo impact of neutralization breadth and sensitivity on prevention efficacy. Such analyses may lead to the validation of a bnAb-based surrogate endpoint for HIV-1 infection, which could accelerate the development of new prevention modalities, such as new bnAb regimens or novel vaccines that induce bnAbs (Liao et al., 2013; Moody et al., 2016; Williams et al., 2017; Zhang et al., 2016). To realize this exciting potential, analyses of randomized trials will need to be informed by in vitro analyses of bnAb breadth and potency. For example, in preparation for the sieve analysis of the AMP trials, Magaret et al. developed models predicting VRC01 neutralization sensitivity using Env amino acid (AA) sequence features (Magaret et al., 2019), based on HIV-1 gp160 pseudoviruses from the Compile, Analyze and Tally NAb Panels (CATNAP) database (Yoon et al., 2015). The super learning ensemble machine learning approach (van der Laan et al., 2007) used to predict rightcensored $50 \%$ inhibitory concentration titer $\left(\mathrm{IC}_{50}\right)$ for each pseudovirus yielded a cross-validated area under the ROC curve (AUC) of 0.868. Magaret et al. also identified important sequence features for predicting VRC01 sensitivity with the goal to enable the AMP sieve analysis to focus on top-ranked features, thereby improving statistical power. Recently, Bricault et al. conducted similar variable importance signature analyses that generalized to all bnAbs across four antibody classes (Bricault et al., 2019).

Given the movement towards combination or multi-specific bnAb regimens, we developed Super LeArner Prediction of NAb Panels (SLAPNAP), a publicly available, containerized pipeline that can perform an end-to-end analysis of in vitro neutralization data for bn $\mathrm{Ab}$ combinations. SLAPNAP analyses of in vitro neutralization data can be used to inform the down-selection of combination or multi-specific bnAb regimens for future efficacy trials and in 
vivo analyses. SLAPNAP leverages all data available in the CATNAP database, and given a user-selected bnAb combination and neutralization endpoint, performs a suite of machine learning-based analyses and provides a report that summarizes the predictive results and highlights important pseudovirus sequence features.

\section{Methods}

SLAPNAP is based on in vitro neutralization data available in the CATNAP database. Predictive analysis of neutralization sensitivity can be done for any single bnAb available in this database, or for combinations of any number of available bnAbs. In the latter case, the neutralization sensitivity of a pseudovirus to the combination bnAb regimen is estimated based on a Bliss-Hill model (Wagh et al., 2016). The models created by SLAPNAP will predict one of three continuous measures of sensitivity [estimated $\mathrm{IC}_{50}$, estimated $\mathrm{IC}_{80}$, and instantaneous inhibitory potential (IIP) (Shen et al., 2008)] and/or two binary measures of sensitivity [whether the estimated $\mathrm{IC}_{50}<$ a user-specified cutpoint, and whether the estimated $\mathrm{IC}_{50}<$ the cutpoint for a user-specified number of bnAbs in the specified combination (multiple sensitivity)]. Predictive models can be built using random forests (Breiman, 2001), boosted regression trees (Friedman, 2001), elastic net (Zou and Hastie, 2005), and/or a super learner ensemble (van der Laan et al., 2007). Cross-validated tuning parameter selection is also available. Feature importance is estimated using the methods of Williamson et al. (Williamson et al., 2020a; Williamson et al., 2020b) and algorithm-specific importance measures (e.g., coefficient magnitude for the elastic net) can also be reported.

\section{Usage}


SLAPNAP is a Docker container hosted on DockerHub (Docker Inc., 2019). With Docker installed, SLAPNAP can be downloaded by executing the following at the command line:

docker pull slapnap/slapnap:latest

SLAPNAP is executed using the docker run command. For example, the following code will instruct SLAPNAP to create and evaluate a neutralization predictor for the bnAb combination VRC07-523-LS and PGT121:

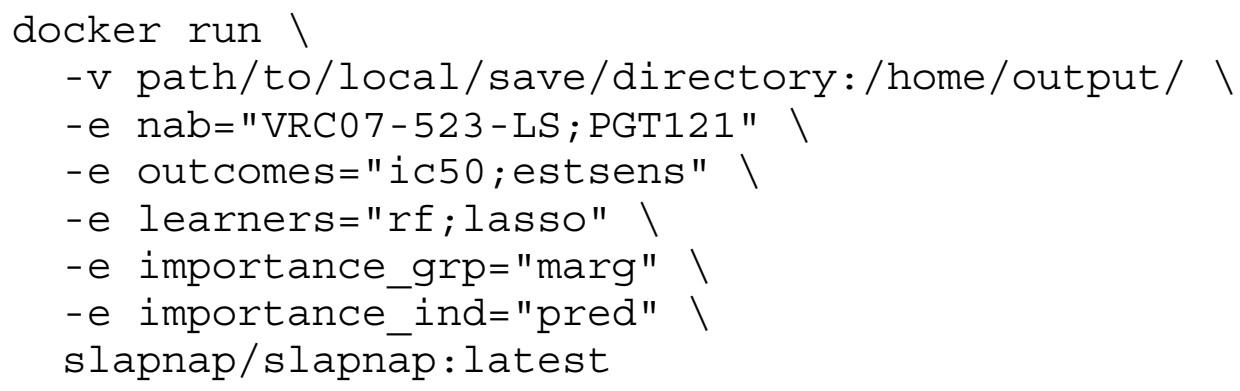

The $-\mathrm{v}$ tag specifies the directory on the user's computer where the report will be saved, and path/to/local/save/directory should be replaced with the desired target directory. Options for the analysis are passed to the container via the -e tag; these options include the bnAbs to include in the analysis (nab), the neutralization outcomes of interest (outcomes), the learners to use in the analysis (learners), and the types of variable importance to compute (importance_grp, for groups of variables; importance_ind, for individual variables). Other output (e.g., the formatted analysis dataset and the fitted learners) can be requested via the return option. A full list of options and their syntax are available in the SLAPNAP documentation (https://benkeser.github.io/slapnap/).

Following the completion of a SLAPNAP run, the HTML report - along with any other requested output — will be saved to the output directory and the SLAPNAP container will shut down. Excerpts from the report are shown in Figure 1. Each report begins with a section 
summarizing data extraction and key results (Panel A). Descriptive statistics and plots are provided for each requested bnAb and for the estimated sensitivity measures for the combined bnAbs (Panel B). The performance of the learners for predicting binary sensitivity endpoints is estimated using cross-validated ROC analysis (Panel C). Feature importance is determined by a variety of methods and summarized in tables and figures (Panel D).

A

\section{Executive summary}

The broady neutralizing antbodies (bNAbs) studied in this analysis are VRC07-523-LS and PGT121. The analysis considered 2 measures of neutralization sensitivity. IC $\mathrm{s}_{0}$ and estimated sensitivity. Estimated IC was computed based on the additive model of Wagh et a!, (2016); for $J$ bNabs, it is computed as

$$
\text { estimated IC }=\left(\sum_{j=1}^{\prime} \mathrm{IC}_{j}^{-1}\right)^{-1} .
$$

Where $I_{C}$, denotes the measured IC $\mathrm{C}_{50}$ for $\mathrm{BNAb} j$. Estimated sensitivity is defined by the binary indicator that estimated IC $\mathrm{C}_{S_{0}}<1$. Based on this specification of bNAbs and outcomes:

- 402 sequencas were extracted from the CATNAP database (Noon et al, 2015)

- 401 sequences had complete geographic and genetic sequence information;

- 401 of these sequences had measured $\mathrm{IC}_{s_{0}}$ :

- out of the sequences with complete data, 378 were estimated to be senstive to the combination of bNAbs, while 23 were estimated to be resistant.

Prediction of each outcome was performed using a super learner ensemble (van der Laan, Polley, and Hubbard 2007) of random forest (Breiman 2001) and elastic net regression (Zou and Hastie 2005) and

C intercept-only regression.

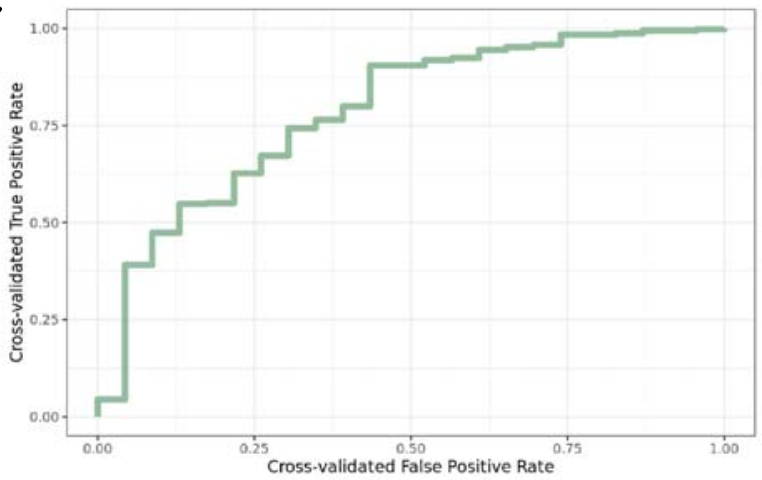

B
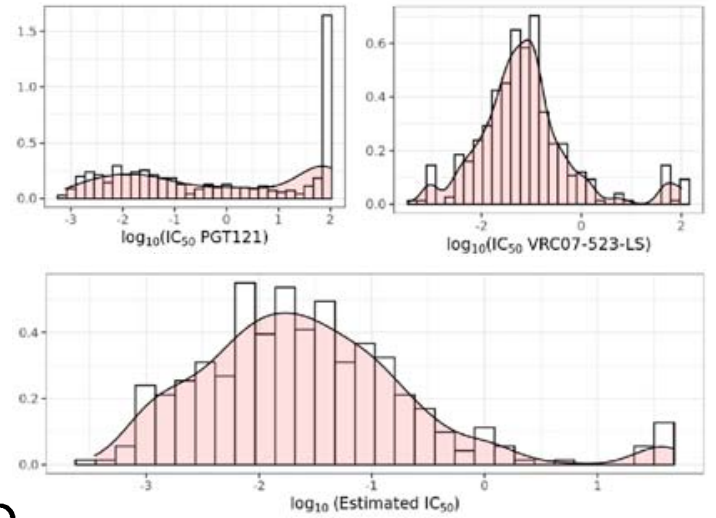

D

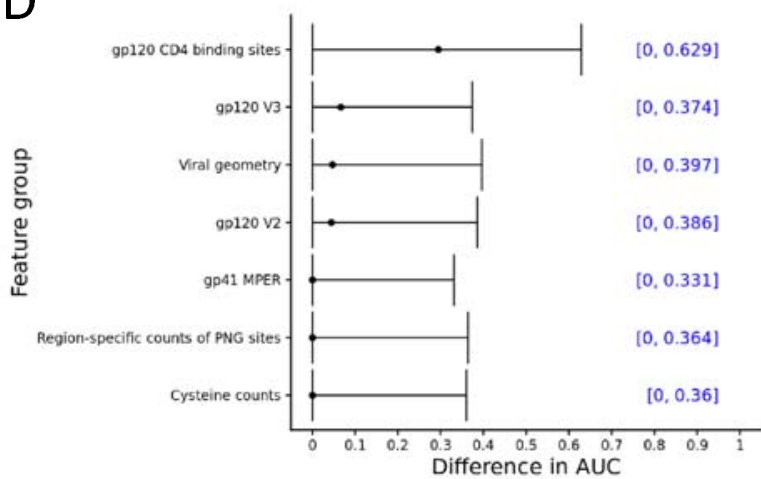

\section{Funding}

This work was supported by the National Institute of Allergy and Infectious Diseases of the

National Institute of Health [grant number UM1 AI068635 (HVTN SDMC) to P.B.G.].

\section{Acknowledgements}

Conflict of interest: None declared. 


\section{References}

Breiman, L. Random forests. Mach Learn 2001;45(1):5-32.

Bricault, C.A. et al. HIV-1 Neutralizing Antibody Signatures and Application to Epitope-Targeted Vaccine Design. Cell Host Microbe 2019;25(1):59-72 e58.

Docker Inc. Docker Hub Quickstart. Docker Docs. https://docs.docker.com/docker-hub/ Accessed 30 Mar 2020. 2019.

Doria-Rose, N.A. et al. HIV-1 neutralization coverage is improved by combining monoclonal antibodies that target independent epitopes. J Virol 2012;86(6):3393-3397.

Friedman, J.H. Greedy function approximation: a gradient boosting machine. Annals of Statistics 2001:1189-1232.

Gautam, R. et al. A single injection of anti-HIV-1 antibodies protects against repeated SHIV challenges. Nature 2016;533(7601):105-109.

Gilbert, P.B. et al. Basis and Statistical Design of the Passive HIV-1 Antibody Mediated Prevention (AMP) Test-of-Concept Efficacy Trials. Stat Commun Infect Dis 2017;9(1).

Hessell, A.J., Malherbe, D.C. and Haigwood, N.L. Passive and active antibody studies in primates to inform HIV vaccines. Expert Rev Vaccines 2018;17(2):127-144.

Karuna, S.T. and Corey, L. Broadly Neutralizing Antibodies for HIV Prevention. Annu Rev Med 2020;71:329-346.

Kong, R. et al. Improving neutralization potency and breadth by combining broadly reactive HIV-1 antibodies targeting major neutralization epitopes. $J$ Virol 2015;89(5):2659-2671.

Liao, H.X. et al. Co-evolution of a broadly neutralizing HIV-1 antibody and founder virus. Nature 2013;496(7446):469-476.

Magaret, C.A. et al. Prediction of VRC01 neutralization sensitivity by HIV-1 gp160 sequence features. PLoS Comput Biol 2019;15(4):e1006952.

McCoy, L.E. and Burton, D.R. Identification and specificity of broadly neutralizing antibodies against HIV. Immunol Rev 2017;275(1):11-20. 
Moody, M.A. et al. Immune perturbations in HIV-1-infected individuals who make broadly neutralizing antibodies. Sci Immunol 2016;1(1):aag0851.

Morris, L. and Mkhize, N.N. Prospects for passive immunity to prevent HIV infection. PLoS Med 2017;14(11):e1002436.

Pegu, A. et al. A Meta-analysis of Passive Immunization Studies Shows that Serum-Neutralizing Antibody Titer Associates with Protection against SHIV Challenge. Cell Host Microbe 2019;26(3):336346 e333.

Shen, L. et al. Dose-response curve slope sets class-specific limits on inhibitory potential of anti-HIV drugs. Nat Med 2008;14(7):762-766.

Sok, D. and Burton, D.R. Recent progress in broadly neutralizing antibodies to HIV. Nat Immunol 2018;19(11):1179-1188.

van der Laan, M.J., Polley, E.C. and Hubbard, A.E. Super learner. Stat Appl Genet Mol Biol 2007;6:Article25.

Wagh, K. et al. Optimal Combinations of Broadly Neutralizing Antibodies for Prevention and Treatment of HIV-1 Clade C Infection. PLoS Pathog 2016;12(3):e1005520.

Wagh, K. et al. Potential of conventional \& bispecific broadly neutralizing antibodies for prevention of HIV-1 subtype A, C \& D infections. PLoS Pathog 2018;14(3):e1006860.

Williams, W.B. et al. Initiation of HIV neutralizing B cell lineages with sequential envelope immunizations. Nat Commun 2017;8(1):1732.

Williamson, B. et al. Nonparametric variable importance assessment using machine learning techniques Biometrics (In Press) 2020a.

Williamson, B.D. et al. A unified approach for inference on algorithm-agnostic variable importance. arXiv:2004.03683 [stat.ME] 2020b.

Yoon, H. et al. CATNAP: a tool to compile, analyze and tally neutralizing antibody panels. Nucleic Acids Res 2015;43(W1):W213-219. 

available under aCC-BY-NC-ND 4.0 International license.

Zhang, R. et al. Initiation of immune tolerance-controlled HIV gp41 neutralizing B cell lineages. Sci Transl Med 2016;8(336):336ra362.

Zou, H. and Hastie, T. Regularization and variable selection via the elastic net. Journal of the Royal Statistical Society: Series B (Statistical Methodology) 2005;67(2):301-320. 